\title{
Validity and Reliability of the Oral Assessment Guide for Children and Young People Receiving Chemotherapy
}

\author{
Şule ÇifTCioĞLU, ${ }^{1}$ Emine EFE' ${ }^{1}$ \\ 'Department of Akdeniz University Nursing Faculty, Faculty of Nursing, Antalya-Turkey
}

\begin{abstract}
OBJECTIVE
This methodological study aimed to adapt the Oral Assessment Guide for Children and Young People into Turkish and assess its validity and reliability.

\section{METHODS}

The study sample comprised 60 children aged 8-18 years who had received chemotherapy once and had agreed to participate in the study.

RESULTS

Internal consistency was used to determine the reliability of the Turkish version of the scale. Cronbach's alpha coefficient was found to be 0.84 . Content and concurrent validity tests were used to determine the validity of the Turkish scale. The experts' opinions showed that the items on the scale correlated well with each other (items content validity index and scale content validity index, 0.91). The concurrent validity was determined through item analysis based on differences between the mean scores of upper-lower group items. A statistically significant difference was found between the mean scores of the upper and lower groups. All items of the scale were found to have high correlations with each other $(\mathrm{p}<0.05)$.
\end{abstract}

\section{CONCLUSION}

The analyses conducted on the Turkish version of the scale showed that it was consistent with the original scale and was valid and reliable for the Turkish society.

Keywords: Chemotherapy; child; oral assessment; oral mucositis; reliability; validity. Copyright $\odot$ 2017, Turkish Society for Radiation Oncology

\section{Introduction}

The rate of childhood cancer has increased in the last 10 years.[1] The Turkish Statistical Institute reported that cancer was the second most common cause of death in children aged $\geq 5$ years, with a rate of $16.4 \%$. [2] Regarding the children between 0 and 14 years of age suffering from cancer, $35.5 \%$ have leukemia.[3]

Oral mucositis refers to the inflammatory or ulcerative lesions in the oral mucosa of patients with cancer receiving chemotherapy or radiotherapy, which can turn into intensely painful sores from mild rashes. [4-6] Chemotherapy damages the regenerative cells in mucosa and causes saliva to lose its protective feature, facilitating the development of oral mucositis.[7-9] The prevalence of oral mucositis was $42.5 \%$ among inpatients and the admission length was increased with increasing severity. Patient diagnosis, chemotherapy treatment block, day of chemotherapy cycle, and neutropenic status have been shown to influence the risk of developing oral mucositis.[10] Pain, bleeding, ulceration, mouth dryness, change in taste, and malnutrition are generally observed in mucositis. The severity of oral mucositis leads to the tapering or postponement of chemotherapy and a longer period of hospitalization.[11,12] Moreover, it may cause skipping or 


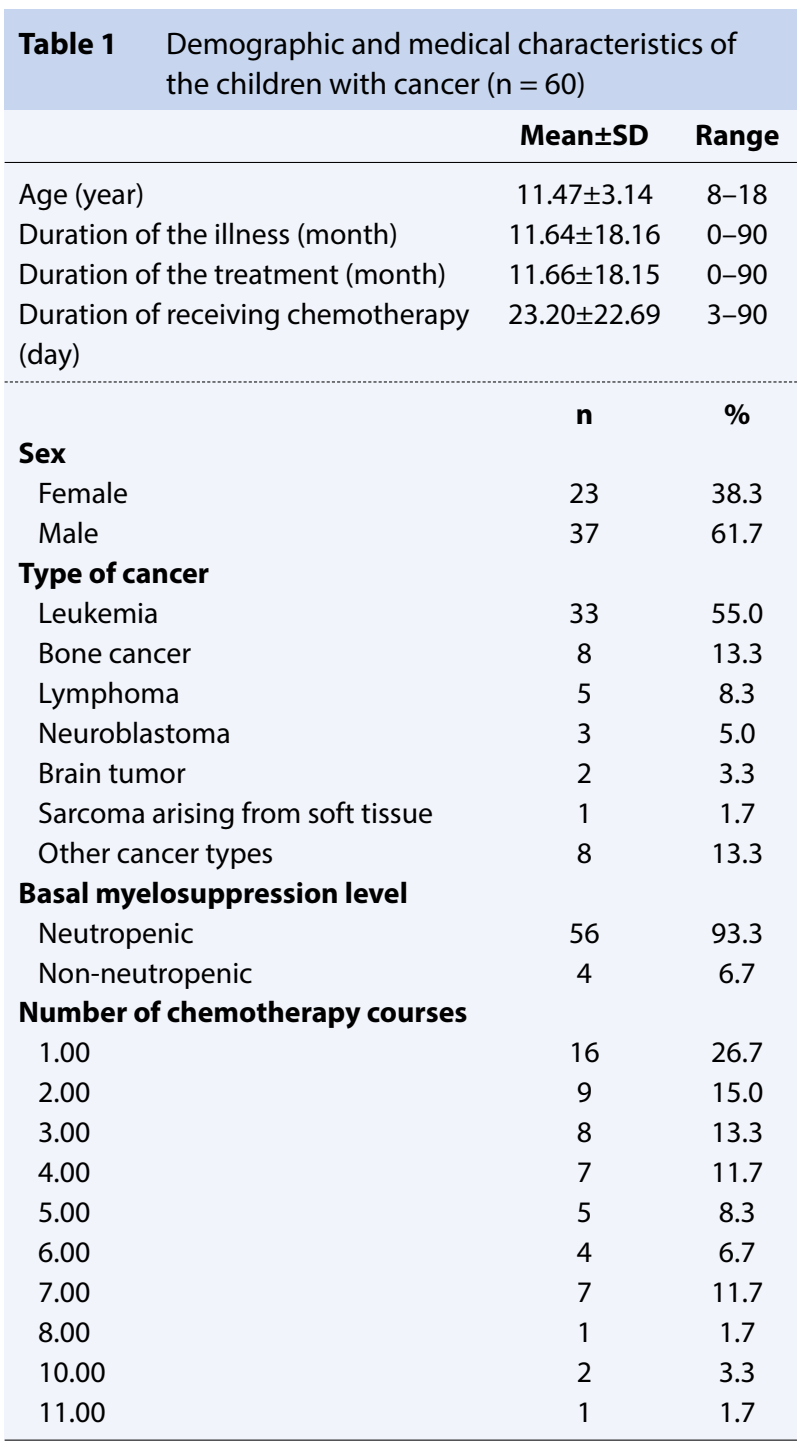

reducing chemotherapy doses. Therefore, long-term therapy results may be negatively affected.[13]

It is important to assess oral mucositis for preventing and ensuring its early diagnosis and treatment if it develops. Easy-to-use, valid, and reliable scales are needed to assess oral mucosa in children. Scales can be used to anatomically, symptomatically, and functionally assess oral mucosa.[14,15] Pediatric oncology nurses play a crucial role in evaluating and promoting oral hygiene and minimizing the potentially debilitating effects of oral mucositis. Most commonly, nurses make decisions regarding oral care. The lack of well-tested and validated instruments also has negative effects on clinical care. Oral assessment may provide baseline data; ensure that oral complications are predicted, and thus prevented or minimized; and enable the evaluation of nursing interventions.[16] A better definition of oral assessment can also s oral status. Structured symptom assessment results in health professionals experience of symptoms.[17]

The scales that are most commonly used for clinical assessment of mucositis include functional scales such as the scale of the World Health Organization (WHO) and the National Cancer Institute Common Toxicity Criteria based on the ability to eat and drink. However, it has been pointed out that $\mathrm{WHO}$ and other functional scales may under-report mucositis if analgesia is given and is effective. [4,8] The National Cancer Institute Common Toxicity Criteria is used to observe the findings of and determine the severity of oral mucositis by identifying its effects on eating function.[4] Another scale that can be used to assess oral mucositis is the Oral Mucositis Assessment Scale.[18] Sung et al. reported that the Oral Mucositis Assessment Scale is valid for use in mucositis clinical trials for children at s International Mucositis Evaluation Scale (ChIMES) was developed to assess oral mucositis in pediatric patients with cancer and the findings related to mucositis.[19] Yavuz et al. adapted ChIMES into Turkish and tested its validity and reliability.[20]

The Oral Assessment Guide (OAG), developed by Eilers et al., was considered to be a useful research tool appropriate for everyday clinical practice use in both adults and children.[21,22] Cheng et al.[23] reported that OAG was effective in the oral assessment of 42 children receiving chemotherapy between 6 and 17 years of age. Cheng et al.[24] administered OAG to 14 children receiving chemotherapy between 6 and 17 years of age at the beginning of chemotherapy and twice a week for the following 3 weeks. They reported that this guide was effective in developing an oral care protocol.

Gibson et al. modified the original scale at Great Ormond Street Hospital for Children. Hence, the abbreviation of this hospital's name was added to the abbreviation of the revised scale and the modified scale was named Oral Assessment Guide for Children and Young People (GOSH OAG). They found the content validity index (CVI) of the modified guide to be 0.83 . The fact that this value was $>0.80$ shows that the scale has a high validity. Gibson et al.[16]reported that the modified scale was appropriate for children and adults. The parameters of GOSH OAG enable nurses to examine oral mucosa in detail. Using a light source for examining the inside of the mouth ensures early diagnosis of mucositis.

Two of the scales developed to assess mucositis in children with cancer were adapted into Turkish: ChIMES [20] and the WHO scale. The Turkish nurs- 


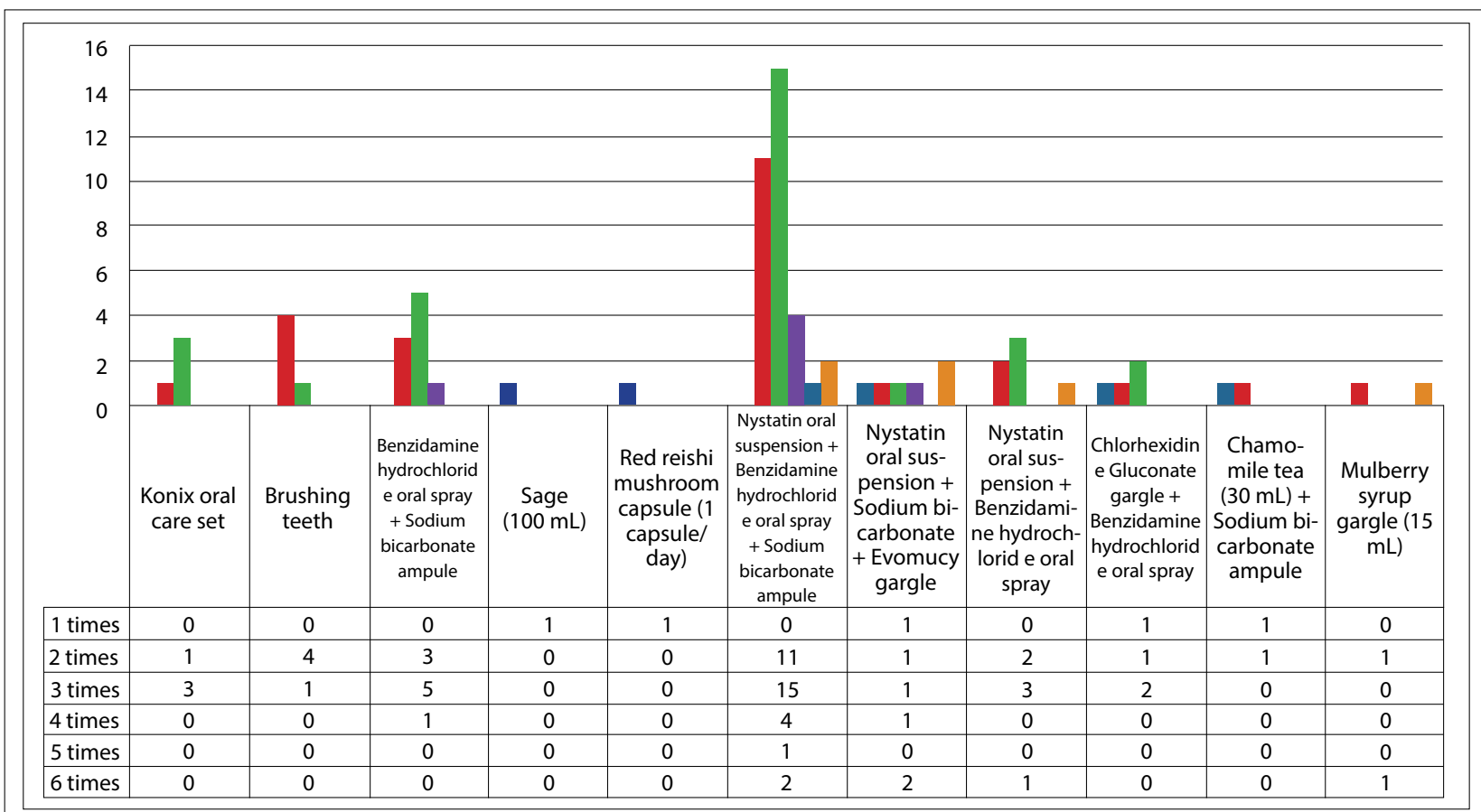

Fig. 1. Frequency of administration of the oral care methods in children with cancer.

es use the WHO scale in clinics to assess mucositis in children with cancer. However, this scale was found to be insufficient for clinical practice. The early diagnosis of mucositis is important for nurses to quickly assess $\mathrm{s}$ oral mucosa in detail. Testing the validity and reliability of GOSH OAG in Turkish is considered to enable the nurses to more quickly and accurately assess the oral mucosa of children with cancer. A new scale is needed in the clinics in Turkey to diagnose mucositis in children with cancer at an early stage.

GOSH OAG has not been translated into several languages yet. No reports were previously put forward on translation or validation of GOSH OAG into Turkish. The Turkish nurses need a scale to assess the inside of the s mouth.

This study aimed to examine whether the Turkish GOSH OAG was a valid and reliable tool to assess mucositis and whether it could be used as a clinical research instrument. This study was conducted to translate GOSH OAG into Turkish and test the reliability and validity of mucositis in children with cancer.

\section{Materials and Methods}

\section{Scale}

GOSH OAG was developed by Gibson et al. It is a scale designed to assess oral status in children following chemotherapy and/or radiotherapy. GOSH OAG consists of the following eight items: (1) voice, (2) swallow, (3) lips and edge of the mouth, (4) tongue, (5) saliva, (6) mucous membranes, (7) gingiva, and (8) teeth.

Each category descriptor is scored from 1 to 3: 1, normal; 2 , mild alterations without severe compromise of either epithelial integrity or systemic functioning; and 3, definite compromise. The eight subscale scores are summed to obtain an overall assessment (minimum, 8; maximum, 24). Higher scores on the scale indicate an increased risk of mucositis or the actual development of mucositis. This guide enables to identify the changes in mucosa at an early stage. GOSH OAG was proven to be clinically useful to assess, record, and communicate oral changes in children and young people who underwent chemotherapy and/or radiotherapy in clinical settings. CVI of the guide was found to be 0.83 , indicating a high level of validity.[16]

\section{Patients, Data Collection, and Statistical Analysis}

The study was planned to adapt GOSH OAG into Turkish language and culture and evaluate the validity and reliability of the Turkish version. The study was conducted in the pediatric oncology and hematology unit of a university hospital in Antalya, Turkey.

According to Esin, the sample size can be taken as 3-10 times the number of items in the inventory.[24] [26] In this study, as the scale had eight items, it was 
Table 2 Demographic and medical characteristics of the children with cancer $(n=60)$ Oral care

\begin{tabular}{lc} 
Oral care & $\mathbf{n}$ \\
\hline Daily oral care & 60 \\
Provided & 0 \\
Not provided & 100 \\
Oral care treatment of the clinic & 0.0 \\
$\quad$ Nystatin oral suspension + Benzidamine hydrochloride oral spray + Sodium bicarbonate ampule & 33 \\
Oral care method administered out of the clinic's oral care treatment & 47.1 \\
Benzidamine hydrochloride oral spray + Sodium bicarbonate ampule & 9 \\
Nystatin oral suspension + Sodium bicarbonate ampule + Evomucy gargle & 12.9 \\
Nystatin oral suspension + Benzidamine hydrochloride oral spray & 8.5 \\
Brushing teeth & 6.5 \\
Konix oral care set & 5 \\
Chlorhexidine Gluconate gargle + Benzidamine hydrochloride oral spray & 5 \\
Complementary oral care method administered out of the clinic's oral care treatment & 4 \\
Sage (100 mL) & 4 \\
Red reishi mushroom capsule (1 capsule/day) & 5.7 \\
Chamomile tea (30 mL) + Sodium bicarbonate ampule & 5.7 \\
Mulberry syrup gargle (15 mL) & 1 \\
\hline
\end{tabular}

administered to a total of 60 people who agreed to participate in the study. A total of 83 children with cancer who had been receiving medical therapy in the pediatric hematology oncology unit between September 2014 and July 2015 were approached and 60 (89.6\%) of them agreed to participate in the study.

All participants were asked to provide informed consent. A personal data form and GOSH OAG were completed by each participant. Data were collected by the authors during face-to-face interviews. A detailed medical history was obtained for all patients. Information about diagnosis and treatment was noted from the medical records. All patients were assessed in the patient room of the children hematology oncology unit. Patients were explained the aim and study protocol. The scales were administered to patients after obtaining their written consent.

\begin{tabular}{|c|c|c|}
\hline $\begin{array}{l}\text { GOSH OAG item } \\
\text { Cronbach alpha }\end{array}$ & $\begin{array}{l}\text { otal score cor } \\
\text { oefficients }\end{array}$ & relations and \\
\hline Category & Correlation & Cronbach alpha \\
\hline Voice & 0.65 & 0.81 \\
\hline Swallow & 0.80 & 0.78 \\
\hline Lips and edge of the mouth & 0.54 & 0.82 \\
\hline Tongue & 0.54 & 0.82 \\
\hline Saliva & 0.46 & 0.83 \\
\hline Mucous membranes & 0.59 & 0.81 \\
\hline Gingivae & 0.54 & 0.82 \\
\hline Teeth & 0.43 & 0.83 \\
\hline Total Cronbach alpha & & 0.84 \\
\hline
\end{tabular}

\section{Data Analysis}

All the data were entered, checked for missing values, and analyzed using SPSS version 20.0 (SPSS Inc., Chicago, IL, USA) statistical programs. Descriptive statistic, mean, median, frequencies, and percentages were used to show the distribution of the personal characteristics, illness-related characteristics, and oral care practices. Factor analysis was also performed. s alpha and the Pearson correlation test were used to examine the internal reliability and validity, respectively. For all statistical analyses, $\mathrm{p}<0.05$ was considered to be statistically significant.

\section{Ethics Considerations}

Permission to translate and use GOSH OAG into Turkish was granted by the developer Faith Gibson. Permission to conduct this study was received from the authors' institutional ethical committee. The patients were informed about the purpose of the study and what should be expected from them. Participants were assured of the rights of refusal to participate in or to withdraw from the study at any stage without any negative consequences. The anonymity and confidentiality of participants were guaranteed.

\section{Results}

\section{Demographic and Clinical Characteristics}

A total of 60 children with cancer were interviewed in this study. The demographic and clinical characteristics of the participants were examined. The average 
Table 4 Respondent CVI score for content validity entire instrument

\section{Category}

Number of ratings of 3 or 4

Actual CVI

Voice

Swallow

Lips and edge of the mouth

Tongue

1.00

Saliva

Mucous membranes

Gingiva

Teeth

Voice

Normal

Deeper or raspy

Difficult talking or crying or suffering pain

\section{Swallow}

Normal swallowing

A little pain while swallowing

Unable to swallow, pooling of secretions

\section{Lips and edge of the mouth}

Smooth, pink, and moist

Dry or cracked

Ulcerated or bleeding

\section{Tongue}

Pink, moist, and papilla present

Coated/Loss of papillae; shiny appearance with or without

redness and/or oral candida

Blistered or cracked

\section{Saliva}

Watery, excess saliva due to teething

Thick or ropy

Absent

\section{Mucous membranes}

Pink and moist

Reddened or coated without ulceration and/or oral candida

Ulceration with or without bleeding

\section{Gingivae}

Pink or coral with a stippled surface

Gum margins tight and well defined, no swelling; edema

Spontaneous bleeding

\section{Teeth}

Clean, no debris

Plaque/debris in localized area

Plaque or debris generalized along gum margin

CVI: Content Validity Index

age of the children was 11.47 years [standard deviation $(S D)=3.14$; range $=8-18]$. Of these children, $38.3 \%$ were females and $61.7 \%$ were males. A majority of the participants had leukemia (55.0\%). The average duration of the illness was 11.64 months (SD, 18.16 months), average duration of treatment 11.66 months (SD, 18.15 months), and average duration of chemotherapy 23.20 days (SD, 22.69 days) (Table 1 ).

All children who participated in this study were continuing their oral treatment in the hospital. It was found that $47.1 \%$ of the children were administered nystatin (Fungostatin oral suspension)+benzidamine hydrochlo- 


\begin{tabular}{lccc} 
Table 5 & GOSH OAG simultaneous validity & \\
\hline Groups (N) & Mean \pm SD & t & p \\
\hline Lower group (16) & $1.07 \pm 0.09$ & -17.60 & 0.00 \\
Upper group (16) & $2.33 \pm 0.27$ & & \\
\hline
\end{tabular}

Table 6 Mean scores of the children receiving cancer on GOSH OAG

\begin{tabular}{lcc} 
Items & $\overline{\mathbf{X}}$ & SD \\
\hline Voice & 1.48 & 0.73 \\
Swallow & 1.65 & 0.80 \\
Lips and edge of the mouth & 1.78 & 0.82 \\
Tongue & 1.85 & 0.63 \\
Saliva & 1.60 & 0.81 \\
Mucous membrane & 1.65 & 0.82 \\
Gingiva & 1.37 & 0.64 \\
Teeth & 1.63 & 0.75 \\
Mean & 1.63 & 0.52 \\
\hline SD: Standart Deviation & &
\end{tabular}

ride (Tantum Verde oral spray)+sodium bicarbonate ampule daily (Table 2). The frequency of daily oral care provided to the children receiving chemotherapy (Fig. $1)$.

\section{Internal Consistency of the Turkish GOSH OAG}

GOSH OAG was tested for internal consistency and inter-rater reliability. s alpha internal consistency coefficient was found to be 0.84 in this study. The Cronbach's alpha internal consistency coefficient of the Turkish GOSH OAG was found to be between 0.79 and 0.84 for children with cancer, indicating a satisfactory reliability (Table 3).[25]

\section{Construct Validity of the Turkish GOSH OAG}

CVI for each item of GOSH OAG according to the evaluation of the seven experts. CVI ranged from 0.86 to 1.00 , demonstrating that the items of GOSH OAG highly contributed to the measurement of the oral cavity Angelo et al.[26], CVI of each item descriptors in OAG was also calculated (Table 4).[27]

\section{Criterion Validity/ Concurrent Validity}

The criterion validity of the Turkish GOSH OAG was determined through item analysis based on differences between mean scores of upper-lower group items. Statistically significant differences were found between the mean scores of the upper and lower group items $(t=-17.61, p=0.000)$ (Table 5). Item analysis showed that GOSH OAG had a concurrent validity and could be used to assess inside and around the mouths of children receiving chemotherapy against the risk of oral mucositis.

\section{Mean Scores of Children with Cancer on GOSH OAG} The mean score of children with cancer on the scale was $1.63 \pm 0.52$ on the items scored between 1 and 3 . The children obtained the lowest mean score on the gingiva item $(1.37 \pm 0.64)$ and the highest mean score on the tongue item $(1.85 \pm 0.63)$ (Table 6).

\section{Discussion}

GOSH OAG was first tested for language validity. Language validity criterion was ensured for OAG. The scale was translated by two academic members and two specialists who were fluent in Turkish and English languages.

$s$ ability to accurately measure a feature or variable that it aims to measure s validity is affected by objectivity, distinctiveness, comprehensiveness, easiness to use, and scorability.[28] Generally, three types of validity exist: content validity, criterion validity, and construct validity. This study tested the content validity of OAG. Item and scale validity indices were used for content validity. The opinions of seven experts were obtained to test and evaluate the content validity of GOSH OAG. A consensus was found among the experts regarding the applicability of the items of the scale.[29-31]

$s$ alpha coefficient was used to determine the reliability of GOSH OAG. The s alpha coefficient is a criterion for internal consistency of scale items. Higher $\mathrm{s}$ alpha coefficient indicates higher consistency between the items of a $s$ alpha coefficient of GOSH OAG was found to be 0.84 in this study. Gibson s alpha coefficient of GOSH OAG to be 0.84 in their study.[16] The result of this study was similar to the results obtained by Gibson et al. This study showed that the Turkish GOSH OAG was highly reliable.

It also analyzed the total-item correlation for reliability analysis of GOSH OAG. An acceptable coefficient of $\geq 0.30$ was recommended for the interpretation of totalitem correlation.[32] The total-item score coefficients of OAG were found to be positive and strong in this study, ranging between 0.43 and 0.80 .

All experts in this study agreed on the importance of the eight categories of GOSH OAG, which was confirmed by the high score of CVI per item, ranging from 0.85 to 1.00. The experts also appreciated the scale for its clarity, wording, efficiency, and simplicity. This is essential for an instrument to be used in clinical practice and research. [33] In the original CVI to be high (ranging from 
0.78 to 1) [16], which was in parallel with the finding of this study.

All items of the Turkish scale sufficiently correlated with the total score and had a high level of item reliability. Total-item score analysis was also accepted as an indicator of validity. It also reflected the construct validity of the scale.[32,33]

The literature includes a limited number of studies that aim to develop oral assessment scales for children with cancer who receive chemotherapy.[15,16,19,34] Only one study is available in Turkey regarding the oral assessment scales for children with cancer who receive chemotherapy.[20] With the verification of the validity and reliability of GOSH OAG, an important assessment tool was introduced in Turkey for oral assessment aimed at children with cancer.

\section{Limitations of the Study}

A total of 83 patients who met the inclusion criteria were included in the study; 23 patients did not agree to participate. The major reasons for refusing to participate in the study were as follows: (1) too weak to be interviewed, (2) lack of interest, and (3) wish to withdraw from the study during the data collection using a video camera .

\section{Conclusion}

The Turkish GOSH OAG showed statistically acceptable levels of reliability and validity. Based on psychometric properties, the Turkish GOSH OAG was proven to be a culturally appropriate tool for children with cancer.

Turkish researchers and health care providers can use GOSH OAG to assess mucositis in children with cancer. These are relevant characteristics for the possible use of this simple, applicable questionnaire in assessing patients with cancer who need additional medical management. The authors of this study believed that this instrument would become useful in the future cross-cultural studies on children with cancer. Studies examining the validity of this tool for longitudinal data from clinical samples should be conducted with a larger sample size, including people from different regions in Turkey and diverse populations around the world.

\section{Relevance to Clinical Practice}

Valid measurement instruments are needed to assess mucositis in patients with cancer. Turkish researchers and health care providers can use GOSH OAG, an easyto-use tool, to assess mucositis in children with cancer. GOSH OAG will enable nurses to assess mucositis in children with cancer.

\section{Disclosures Statement}

The authors declare that they have no conflict of interests.

Acknowledgments: The authors wish to thank the authors of GOSH OAG for their permission to translate and use the guide. The authors are especially grateful to all pediatric patients with cancer who participated in this study. They also wish to thank all experts for translation and back-translation and for conceptual and content identification. They are also grateful to Prof. Dr. Mehmet Ziya Firat for statistical consultation. This study has been produced from the Master's thesis and received no financial support for the research and/or authorship of this article.

Ethics Committee Approval: This study was conducted inaccordance with local ethical rules.

Peer-review: Externally peer-reviewed.

Conflict of Interest: None declared.

Authorship contributions: Concept - E. E, Ş. Ç; Design - E. E, Ş. Ç; Supervision - E. E; Materials - Ş. Ç; Data collection \&/ or processing - Ş. Ç; Analysis and/or interpretation - E. E, Ş. Ç; Literature search - Ş. Ç; Writing - E. E, Ş. Ç; Critical review - E. E, Ş. Ç

\section{References}

1. Key Statistics for Childhood Cancers. Available at: http://www.cancer.org/cancer/cancerinchildren/detailedguide/cancer-in-children-key-statistics. Accessed May 15, 2017.

2. Turkish Statistical Institute. Death Statistics Provincial and District Centers 2008. Ankara: Turkish Statistical Institute Press; 2009. p. 25.

3. Turkey Cancer Statistics. Ankara: Turkish Public Health Institution; 2017. p. 22.

4. Peterson DE, Bensadoun RJ, Roila F; ESMO Guidelines Working Group. Management of oral and gastrointestinal mucositis: ESMO Clinical Practice Guidelines. Ann Oncol 2011;22 Suppl 6:vi78-84.

5. Yeaney MN, Chandler MH, Scarbalis KA. Cellular alterations. In: Potts NL, Mandleco BL, editors. Pediatric Nursing Caring for Children and Their Families. 3rd ed. USA: Delmar; 2012. p. 1023-77.

6. Botti S, De Cocco V, Galgano L, Gargiulo G, Magarò A, Orlando L. Oral mucositis in hematopoietic stem cell transplantation (HSCT): position statement by Gruppo Italiano Trapianto di Midollo Osseo (GITMO) Nurses Group. Drugs and Cell Therapies in Hematology 2014;4:205-23. 
7. Oeschger Schürch F, Verdan C. Oncological nursing care. In: Imbach P, Kühne T, Arceci R, editors. Pediatric Oncology: A Comprehensive Guide. Germany: Springer-Verlag; 2006. p. 206-26.

8. Sevinir B. Mucositis. In: Özkan A, editor. Pediatric Oncology. Istanbul: Nobel Medicine Bookstores; 2009. p. 1283-300.

9. Cheng KK, Chang AM, Yuen MP. Prevention of oral mucositis in paediatric patients treated with chemotherapy; a randomised crossover trial comparing two protocols of oral care. Eur J Cancer 2004;40(8):1208-16.

10. Allen G, Logan R, Revesz T, Keefe D, Gue S. The Prevalence and Investigation of Risk Factors of Oral Mucositis in a Pediatric Oncology Inpatient Population; a Prospective Study. J Pediatr Hematol Oncol 2017 Oct 17 [Epub ahead of print], doi: 10.1097/MPH.0000000000000970.

11. Çubukçu ÇE. Oral mucositis and oral health in pediatric cancer patients. Current Pediatrics Journal 2005;3:67-9.

12. Tassinari D, Maltoni M. Gastrointestinal problems. In: Catane R, Cherny NI, Kloke M, Tanneberger S, Schrijvers D, editors. European Society for Medical Oncology Handbook of Advanced Cancer Care. USA: Taylor \& Francis; 2006. p. 49-68.

13. Yllmaz MÇ. Evidence-Based Practices for Managing Mucositis. International Journal of Hematology and Oncology 2007;4(17):241-6.

14. Eilers J, Epstein JB. Assessment and measurement of oral mucositis. Semin Oncol Nurs 2004;20(1):22-9.

15. Sung L, Tomlinson GA, Greenberg ML, Koren G, Judd P, Ota S, et al. Validation of the oral mucositis assessment scale in pediatric cancer. Pediatr Blood Cancer 2007;49(2):149-53.

16. Gibson F, Cargill J, Allison J, Begent J, Cole S, Stone J, et al. Establishing content validity of the oral assessment guide in children and young people. Eur J Cancer 2006;42(12):1817-25.

17. Collins JJ, Byrnes ME, Dunkel IJ, Lapin J, Nadel T, Thaler HT, et al. The measurement of symptoms in children with cancer. J Pain Symptom Manage 2000;19(5):36377.

18. Maloney AM. Gastrointestinal tract. In: Tomlinson D, Kline NE, editors. Pediatric Oncology Nursing Advanced Clinical Handbook. 2nd ed. Berlin Heidelberg: Springer-Verlag; 2010. p. 354-8.

19. Tomlinson D, Gibson F, Treister N, Baggott C, Judd $\mathrm{P}$, Hendershot E, et al. Refinement of the Children's International Mucositis Evaluation Scale (ChIMES): child and parent perspectives on understandability, content validity and acceptability. Eur J Oncol Nurs 2010;14(1):29-41.

20. Yavuz B, Bal Yilmaz H, Karaman N. A study of reli- ability and validity for the Turkish version of children's international mucositisis evaluation scale for children with cancer. Turk J Oncol 2011;26(4):157-62.

21. Eilers J, Berger AM, Petersen MC. Development, testing, and application of the oral assessment guide. Oncol Nurs Forum 1988;15(3):325-30.

22. Deiana GM. Oral care in children undergoing HSCT. The European Group for Blood and Marrow Transplantation-Nurses Group; 2011. Available at: https://www. ebmt.org. Accessed Apr 5, 2017.

23. Cheng KK, Molassiotis A, Chang AM, Wai WC, Cheung SS. Evaluation of an oral care protocol intervention in the prevention of chemotherapy-induced oral mucositis in paediatric cancer patients. Eur J Cancer 2001;37(16):2056-63.

24. Cheng KK, Molassiotis A, Chang AM. An oral care protocol intervention to prevent chemotherapy-induced oral mucositis in paediatric cancer patients: a pilot study. Eur J Oncol Nurs 2002;6(2):66-73.

25. Esin MN. Reliability and validity of data collection methods and tools \& data collection tools. In: Erdoğan S, Nahcivan N, Esin MN, editors. Research in Nursing: Process, Practice and Critical. Istanbul: Nobel Medicine Bookstores; 2014. p. 195-232.

26. D’Angelo D, Vellone E, Salvatori C, De Marinis MG, Alvaro R. Validity and reliability of the Italian version of the oral assessment guide. Prof Inferm 2013;66(2):11724.

27. Karakoç FY, Dönmez L. Basic principles in scale development studies. Medical Education World 2014;40: 9-49.

28. Kelecioğlu H, Göçer Şahin S. From past to present validity. Journal of Measurement and Evaluation in Education and Psychology 2014;5(2):1-11.

29. Ergin DY. Validity and reliability in scales. Marmara University Atatürk Education Faculty Journal of Educational Sciences 1995;7:125-48.

30. Karip E. Measurement and Evaluation. Ankara: Pegem A Publishing; 2007. p. 2-15, 52-79.

31. Gözüm S, Aksayan S. Guidance for the adaptation of the intercultural scale II: psychometric properties and intercultural comparison. Journal of Nursing Research Development 2003;5(1):3-14.

32. Polit D, Beck C. Essentials of Nursing Care: Methods, Appraisal and Utilization. 6th ed. Philadelphia: Lippincott Williams and Wilkins; 2006. p. 377-88.

33. Jacobs S, Baggott C, Agarwal R, Hesser T, Schechter T, Judd P, et al. Validation of the Children's International Mucositis Evaluation Scale (ChIMES) in paediatric cancer and SCT. Br J Cancer 2013;109(10):2515-22. 